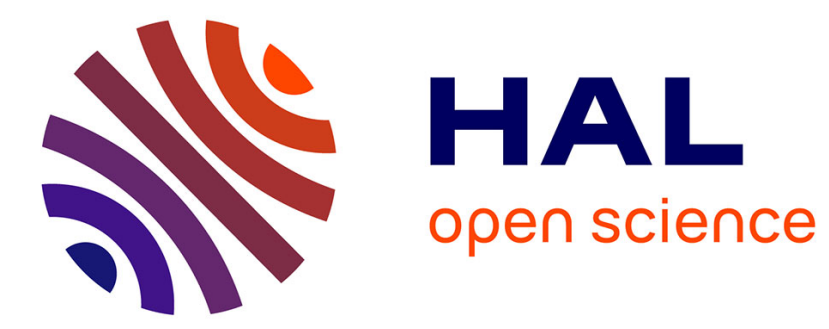

\title{
Staircase scaling of short-time energy transfer in turbulence
}

Le Fang, T. Wu, Wouter J.T. Bos

\section{- To cite this version:}

Le Fang, T. Wu, Wouter J.T. Bos. Staircase scaling of short-time energy transfer in turbulence. Journal of Turbulence, 2020, 10.1080/14685248.2020.1752375 . hal-02549376

\section{HAL Id: hal-02549376 https://hal.science/hal-02549376}

Submitted on 21 Apr 2020

HAL is a multi-disciplinary open access archive for the deposit and dissemination of scientific research documents, whether they are published or not. The documents may come from teaching and research institutions in France or abroad, or from public or private research centers.
L'archive ouverte pluridisciplinaire HAL, est destinée au dépôt et à la diffusion de documents scientifiques de niveau recherche, publiés ou non, émanant des établissements d'enseignement et de recherche français ou étrangers, des laboratoires publics ou privés. 


\title{
Staircase scaling of short-time energy transfer in turbulence
}

\author{
L. Fang, ${ }^{1, *}$ T. Wu, ${ }^{1}$ and Wouter J.T. Bos $^{2}$ \\ ${ }^{1}$ LMP, Ecole Centrale de Pékin, Beihang University, Beijing 100191, China \\ ${ }^{2}$ LMFA, CNRS, Ecole Centrale de Lyon-Université de Lyon, 69130 Ecully, France
}

(Dated: March 24, 2020)

\begin{abstract}
It is illustrated that a sharply truncated initial kinetic energy spectrum evolves to a staircase-shaped spectrum at short times. This effect is directly associated with the triadic nature of the energy transfer. A rigorous analysis leads to predictions on the time-dependence of this effect, and these predictions are verified by both direct numerical simulations and eddy-damped quasi-normal Markovian closure integrations.
\end{abstract}

Keywords: triad interaction; isotropic turbulence; scaling

* Corresponding Author. Email: le.fang@zoho.com 


\section{INTRODUCTION}

The nonlinearity of the Navier-Stokes equations is responsible for the transfer of energy between scales in turbulent flows. The precise understanding and modeling of this transfer is one of the most difficult issues in turbulence research and many theories and models have been developed to represent the energy transfer. For high Reynolds number turbulence, the fundamental framework is the Kolmogorov 1941 theory [29], which associates the forward energy cascade in the inertial range of fully developed turbulence with a conserved flux from the production to the dissipation scales. Dimensional arguments lead then to a prediction of the shape of the kinetic energy spectrum $E(k) \sim k^{-\gamma}$ with $\gamma=5 / 3$, where $k$ is the wavenumber. This scaling is observed with reasonable precision in experiments and simulations [30, 31] (see Ref. [32] for refinements of these arguments).

Obviously in many flow situations the production and dissipation of turbulent kinetic energy are not in equilibrium. A typical academic case is freely decaying turbulence. Experimentally such flows are studied using grid-turbulence in wind-tunnels, and recent investigations have shown that in such experiments the scaling of the dissipation of kinetic energy in the early times, just beyond the production zone, does not concord completely with dimensional arguments associated with Kolmogorov's ideas [33]. Perturbative arguments allow to show that this scaling anomaly is explainable as a first-order perturbation of the Kolmogorov state [34]. Indeed, in the experiments the kinetic energy spectrum is observed to approximately satisfy Kolmogorov scaling. How the spectrum develops before the Kolmogorov scaling is observed is hard to investigate in these experiments, where the near-grid flow is highly statistically inhomogeneous.

Clearly, in grid-turbulence the exact shape of the initial spectrum is hard to control. In numerical experiments, however, turbulent decay can be studied starting from well-designed initial conditions, corresponding to a localized initial spectrum. Such numerical experiments were for instance carried out in the context of the possible determination of vorticity blow-up and singularity formation, where starting from a Taylor-Green vortex, the transient spectrum showed a steep scaling before all spatial scales were excited $[35,36]$. The short-time scaling was further investigated in the context of diffusion models, where it was discovered that a transient scaling was observed with an exponent of the order $1.8<\gamma<1.9$ again steeper than the Kolmogorov value [37]. The same scaling was confirmed by more elaborate closure, 


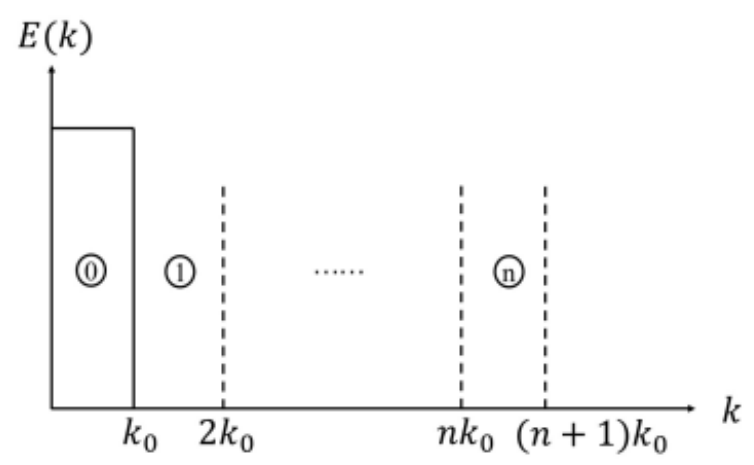

Fig. 1. Definition of the different wavenumber bands of the kinetic energy spectrum. Initially, "band 0" contains all the energy and all other bands are unexcited.

of the eddy-damped quasi-normal Markovian (EDQNM) type [38]. The first appearance of such scaling was observed in magnetohydrodynamic wave-turbulence, where a spectral exponent of $7 / 3$ was observed before the expected $\gamma=2$ inertial slope established [39] and in subsequent wave-turbulence studies this was confirmed [40]. A review of different systems and the scaling predictions from simplified models is given in reference [41].

In the present investigation we will scrutinize the very early development of the kinetic energy spectrum from a spectrally localized initial condition even before power-law scaling is observed. We show that, if the initial condition is not spectrally smooth, the steep gradient from the initial spectrum is not continuously smoothed out, as expected if energy transfer was of purely diffusive nature, but the triadic interactions of the energy transfer causes a stepwisely decreasing energy spectrum at short times. We will then illustrate that this behaviour can be quantitatively predicted. The predictions are assessed using both direct numerical simulations (DNSs) and numerical integration of the EDQNM model.

\section{THEORETICAL ANALYSIS}

In this section we will show how, at very short times, the energy evolves from an initial condition confined to low wavenumbers towards higher wavenumbers. The effect we describe is inherent to the triadic nature of energy transfer in fluid turbulence. Indeed, the quadratic nonlinearity of the Navier-Stokes equations gives rise, in scale space, to the coupling of three scales. In order to understand the inter-scale energy transfer it turns out that the 
Fourier-decomposition is extremely useful, in particular when statistically homogeneous or periodic flows are concerned. In Fourier-space the three-scale or triadic coupling corresponds to the interaction of energy at three different wavevectors, $\boldsymbol{k}, \boldsymbol{p}, \boldsymbol{q}$ which can form a triangle, $\boldsymbol{k}-\boldsymbol{p}-\boldsymbol{q}=0$. This particular triadic interaction will give rise to an irregularity in the short-time energy spectrum when the kinetic energy of the initial condition is confined to the smallest wavenumbers. In order to evaluate the short-time behaviour in an analytical manner, we use the helical mode decomposition which allows, as we will show, to give correct predictions for the evolution of the kinetic energy at short times.

We consider three-dimensional, incompressible, homogenous isotropic turbulence. Following Refs. [42-44], we decompose an incompressible velocity field by using the helical decomposition. This decomposition is a natural one since it takes directly into account the incompressible nature of the flow. The velocity field $\boldsymbol{v}(\boldsymbol{x})$ in physical space is translated to $\boldsymbol{u}(\boldsymbol{k})$ in Fourier space with $\boldsymbol{k}$ the wave vector. Being divergence-free, $\boldsymbol{k} \cdot \boldsymbol{u}(\boldsymbol{k})=0$, each velocity component in Fourier space has only two degrees of freedom. Two orthonormal complex helical waves are chosen to represent the velocity modes, $\boldsymbol{h}^{ \pm}=\hat{\boldsymbol{w}} \times \hat{\boldsymbol{k}} \pm \mathrm{i} \hat{\boldsymbol{w}}$, with $\mathrm{i}=\sqrt{-1}$. The unit vector $\hat{\boldsymbol{w}}$ can be chosen as $\hat{\boldsymbol{w}}=\boldsymbol{z} \times \boldsymbol{k} /\|\boldsymbol{z} \times \boldsymbol{k}\|$ with $\boldsymbol{z}$ an arbitrary vector. We then have

$$
\boldsymbol{u}(\boldsymbol{k})=u^{+}(\boldsymbol{k}) \boldsymbol{h}^{+}(\boldsymbol{k})+u^{-}(\boldsymbol{k}) \boldsymbol{h}^{-}(\boldsymbol{k}) .
$$

Using this decomposition, the Fourier-transform of the Navier-Stokes equation yields

$$
\left(\partial_{t}+\nu k^{2}\right) u^{s_{k}}(\boldsymbol{k})=-\frac{1}{4} \sum_{\boldsymbol{k}+\boldsymbol{p}+\boldsymbol{q}=0} \sum_{s_{p}, s_{q}}\left(s_{p} p-s_{q} q\right)\left[\boldsymbol{h}^{s_{p}} \times \boldsymbol{h}^{s_{q}} \cdot \boldsymbol{h}^{s_{k}}\right] \bar{u}^{s_{p}}(\boldsymbol{p}) \bar{u}^{s_{q}}(\boldsymbol{q}),
$$

where $\nu$ is viscosity, $\boldsymbol{\bullet}$ stands for complex conjugate. The superscripts $s_{k}, s_{p}, s_{q}= \pm$ denote the six different helical modes involved in a triad. When $s_{k}, s_{p}, s_{q}$ are appearing as variables in the expressions, their values are \pm 1 .

We concentrate on the dynamics of an isolated triad, assuming that energy transfer only occurs among three wave numbers $(\boldsymbol{k}, \boldsymbol{p}, \boldsymbol{q})$ with specific helical modes $\left(s_{k}, s_{p}, s_{q}\right)$ respectively. In the inviscid case, this leads to three ordinary differential equations (ODEs) in the complex domain

$$
\begin{aligned}
& \dot{u}^{s_{k}}(\boldsymbol{k})=g\left(s_{p} p-s_{q} q\right) \bar{u}^{s_{p}}(\boldsymbol{p}) \bar{u}^{s_{q}}(\boldsymbol{q}), \\
& \dot{u}^{s_{p}}(\boldsymbol{p})=g\left(s_{q} q-s_{k} k\right) \bar{u}^{s_{q}}(\boldsymbol{q}) \bar{u}^{s_{k}}(\boldsymbol{k}), \\
& \dot{u}^{s_{q}}(\boldsymbol{q})=g\left(s_{k} k-s_{p} p\right) \bar{u}^{s_{k}}(\boldsymbol{k}) \bar{u}^{s_{p}}(\boldsymbol{p}),
\end{aligned}
$$


with $\bullet$ time derivative, and $g=-1 / 4\left[\boldsymbol{h}^{s_{p}} \times \boldsymbol{h}^{s_{q}} \cdot \boldsymbol{h}^{s_{k}}\right]$.

We consider an initial spectral energy distribution truncated at wavenumber $k_{0}$ by a spectral cutoff filter. This band which initially contains all the energy is denoted as "band 0", as shown in Fig. 1. In the figure the energy spectrum is assumed to be uniform in band 0, but a non-uniform distribution will not qualitatively affect the result. In order to describe the small-scale response scaling, we denote the band between $n k_{0}$ and $(n+1) k_{0}$ as "band $n "$, with $n$ a positive integer. If $k$ is located in band $n$, the energy density $e_{n}(k, t)$ is defined as $e_{n}(k, t)=\frac{1}{4 \pi k^{2}} \int_{|\boldsymbol{k}|=k}\left(u^{+}(\boldsymbol{k}, t)^{2}+u^{-}(\boldsymbol{k}, t)^{2}\right) d \boldsymbol{k}$.

Now that we have defined the helical decomposition and the discretization of wavenumber space, we will show how the kinetic energy evolves under the influence of the isolated triad dynamics (3). To do this, we have the following proposition that we will prove by mathematical induction.

Proposition 1. For a fixed wave vector $\boldsymbol{k}$ with wavenumber $k=|\boldsymbol{k}|$ located in band $n$, the magnitude of the helical mode $u_{n}^{s_{k}}(\boldsymbol{k}, t)$ is of order $t^{n}$ for small time $t$, and the energy density $e_{n}(k, t)$ is thereby of order $t^{2 n}$

Proof. i) Illustrating that for $n=1$ Proposition 1 is satisfied. At the initial momen$\mathrm{t}$, for a fixed wave vector $\boldsymbol{k}$ located in band 1, all triad interactions involve exclusively modes with the wave vectors located in band 0, denoted $(\boldsymbol{k}, \boldsymbol{p}, \boldsymbol{q})$. From Eq. (3), we can obtain $\left.\dot{u}^{s_{k}}(\boldsymbol{k}, t)\right|_{t=0} \neq 0$ when $s_{p} p \neq s_{q} q$. (Note that $s_{p} p=s_{q} q$ is a special case where $\dot{u}^{s_{k}}(\boldsymbol{k}, t), \dot{u}^{s_{p}}(\boldsymbol{p}, t), \dot{u}^{s_{q}}(\boldsymbol{q}, t)$ are all zero and there is no energy transfer among the three modes). At short times, $\dot{u}^{s_{k}}(\boldsymbol{k}, t)$ can therefore be considered as a nonzero constant. Besides, there is no energy in band 1 at the initial moment, thus $\left.u^{s_{k}}(\boldsymbol{k}, t)\right|_{t=0}=0$. Therefore, for a triad with two legs $(\boldsymbol{p}, \boldsymbol{q})$ in band zero and one $(\boldsymbol{k})$ in band 1 , the first equation of (3) gives,

$$
\dot{u}^{s_{k}}(\boldsymbol{k})=\text { Constant }
$$

so that $u_{1}^{s_{k}}(\boldsymbol{k}, t) \sim O(t)$ for small time t. From the definition of energy density, we obtain $e_{1}(k, t) \sim O\left(t^{2}\right)$ for small time t. Proposition 1 is satisfied.

ii) Inductive step:

Assuming that Proposition 1 is satisfied for all $n \leqslant m$ with $m$ positive integer greater than 1 , we will prove the propositions for $m+1$. Without losing gener- 
ality, we assume that for a triad interaction $(\boldsymbol{k}, \boldsymbol{p}, \boldsymbol{q})$, wave vector $\boldsymbol{k}$ is located in band $m+1, \boldsymbol{p}$ in band $a$, and $\boldsymbol{q}$ in band $b$, with $a$ and $b$ positive integers smaller than, or equal to $m$. For Proposition 1, we can obtain that in a short period of time $u^{s_{p}}(\boldsymbol{p}, t) \sim O\left(t^{a}\right)$ and $u^{s_{q}}(\boldsymbol{q}, t) \sim O\left(t^{b}\right)$. From Eq. (3), we can obtain $\dot{u}^{s_{k}}(\boldsymbol{k}, t) \sim O\left(t^{a+b}\right)$ in this triad interaction. By the triangle inequality, the lengths of these three wavevectors must satisfy $p+q \geqslant k$ which implies $(a+1)+(b+1) \geqslant(m+1)+1$, and therefore $a+b \geqslant m$. This means that the contribution from a single triad interaction $(\boldsymbol{k}, \boldsymbol{p}, \boldsymbol{q})$ to the energy growth at $\boldsymbol{k}$ is of the order $t^{a+b} \gtrsim t^{m}$. From Eq. (2), $\dot{u}^{s_{k}}(\boldsymbol{k}, t)$ is the summation of all these triad interactions, thus $\dot{u}^{s_{k}}(\boldsymbol{k}, t) \sim O\left(t^{m}\right)$. Therefore, $u_{m+1}^{s_{k}}(\boldsymbol{k}, t) \sim O\left(t^{m+1}\right)$ for small time $t$. From the definition of energy density, we can obtain $e_{m+1}(k, t) \sim O\left(t^{2(m+1)}\right)$ for small time $t$. Proposition 1 is thereby satisfied.

Therefore, Proposition 1 is satisfied for all $n \in \mathbb{N}^{+}$.

We remark that Proposition 1 is a general result for the scaling of short-time energy transfer. However, the growth rate can be affected by phase correlations and the helical properties of the velocity modes [44-46] constituting the initial field. For instance, we can imagine a single-scale initial velocity field constructed by homochiral modes (i.e., $s_{p}=s_{q}$ ), and $u^{s_{k}}(\boldsymbol{k})$ is the same for all wave vectors $\boldsymbol{k}$ with the same length $k$. It is easy to show that this (perhaps somewhat pathological) case is a fixed point of the Euler equation corresponding to zero energy transfer. Such initial conditions will lead to slow initial transfer of energy.

\section{NUMERICAL RESULTS}

In the foregoing section we have argued that the energy density $e_{n}(k, t)$ in band $n$ will evolve proportional to $t^{2 n}$. In this section, we will assess this using DNS and EDQNM, respectively.

\section{A. DNS results}

DNSs are performed using a standard pseudo-spectral solver and a fourth-order RungeKutta time integration scheme, with a semi-implicit treatment of the viscous term [47]. The computational domain has $256^{3}$ grid points, with domain size $(2 \pi)^{3}$, so that the minimum wavenumber is $k_{\min }=1$. A hyper-viscous term of the form $\nabla^{4} \boldsymbol{v}$ is used to increase the 


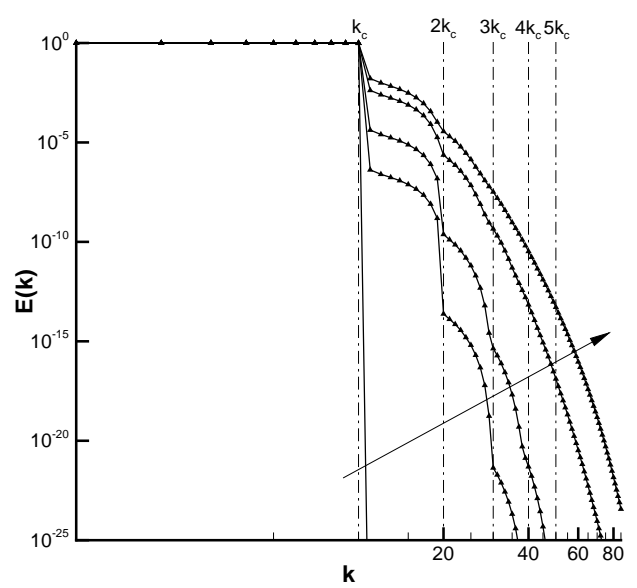

(a)

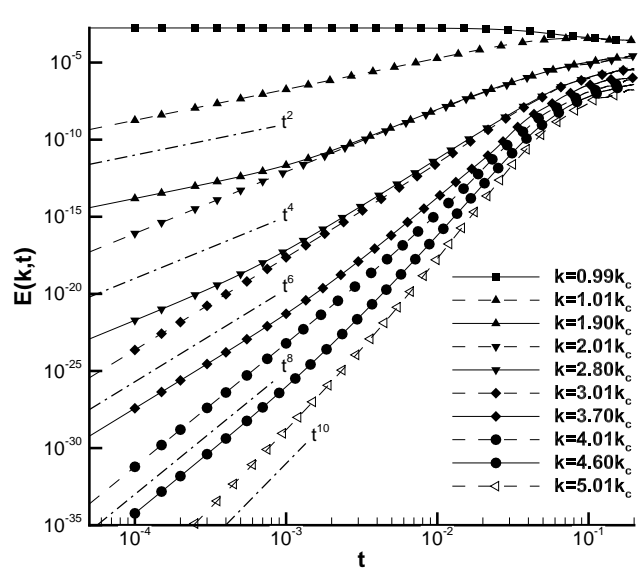

(b)

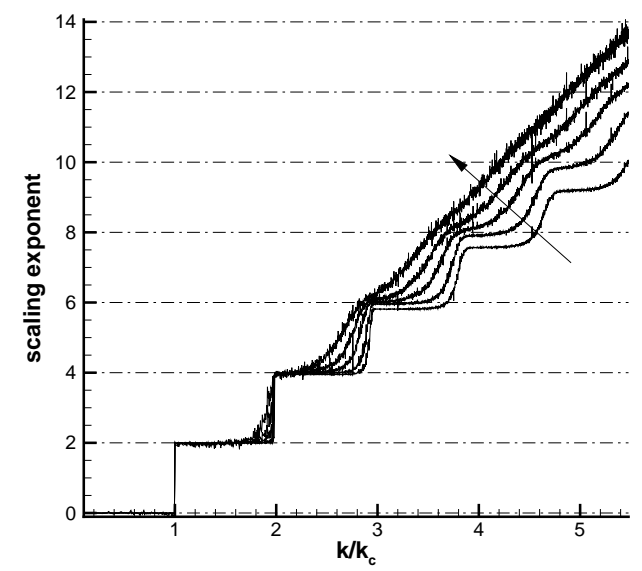

(c)

Fig. 2. (a) Energy spectra at different time in the DNS case. Curves correspond to $t=$ $0,0.00005,0.0005,0.005$ and 0.01 respectively. The arrow indicates the direction of time. (b) Energy evolution at given wavenumbers in the DNS case. Curves with the same symbol are located in the same energy band. (c) Temporal scaling exponent at different times (chosen as in Fig. (a)).

size of the inertial range for a given resolution. Rogallo's method[48] is used to generate Gaussian initial field with random phase correlations. We remark that different initial phase correlations may slow down or accelerate the energy transfer, but the staircase scaling should not change. The band width is selected as $k_{c}=10$, indicating that the ratio between the lengths of the longest and shortest legs in a triad can be upto 10. This is to guarantee that 


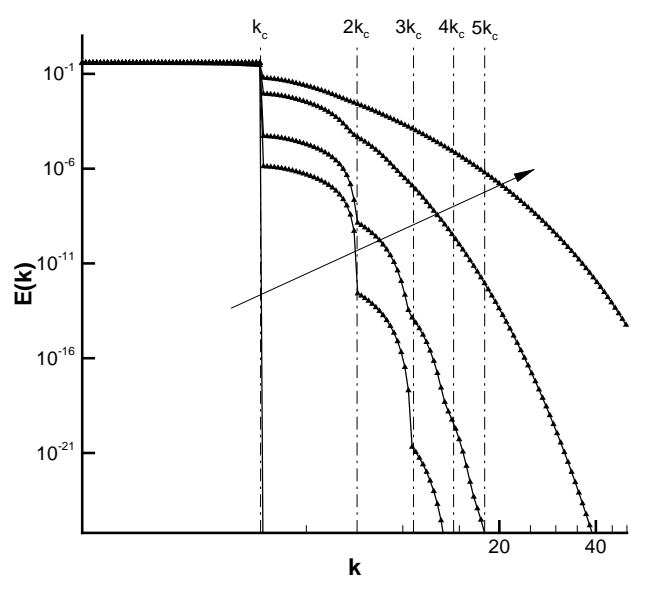

(a)

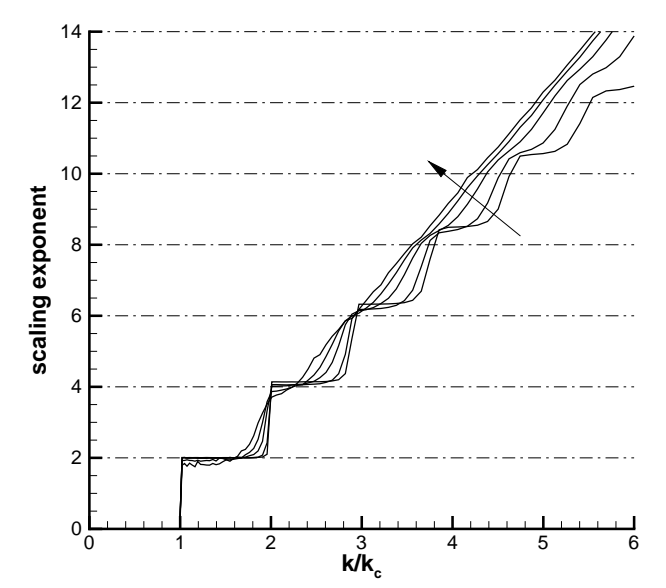

(b)

Fig. 3. (a) Energy spectra at different time in the EDQNM case. Curves correspond to $t=$ $0,0.00088,0.0067,0.072$ and 0.144 respectively. The arrow indicates the direction of time. (b) Temporal scaling exponent at different times (chosen as in Fig. (a)).

the DNS case is consistent to the theoretical analysis, where both local and non-local triad interactions contribute to the staircase scaling $[49,50]$.

The energy spectra $E(k)$, defined as the integral of energy density $e(k)$ over a sphere with radius $k$, are shown in Fig. 2(a) at short times. Clearly, there are discontinuities in the spectral slope at the boundaries of each band, denoted as vertical lines in the figure. We then select wavenumbers around these band boundaries, and show their energy evolution in Fig. 2(b). It is found that wavenumbers in the same band obey the same time scaling in the very beginning of the simulation. As an example, when $k=1.90 k_{c}$, energy increases as $t^{2}$, whereas this scaling is proportional to $t^{4}$ for $k=2.01 k_{c}$. In order to quantitatively examine these results, we define the local time-scaling exponent $n(k, t)$ as

$$
n(k, t)=\frac{\partial E(k, t)}{\partial t} \frac{t}{E(k, t)},
$$

which is shown in Fig. 2(c). Staircase time scalings are clearly observed. At higher wavenumbers the wavenumber dependence of the scaling exponent smoothens rapidly, since the spectrum is less sharply truncated there. The reason is that there are few triad combinations to transfer energy to the largest wavenumbers. 


\section{B. EDQNM results}

We also integrate the EDQNM closure to assess the small-scale response. The closed equation for $E(k)$ reads [51, 52],

$$
\left(\frac{\partial}{\partial t}+2 \nu k^{2}\right) E(k, t)=\iint_{\Delta} \theta_{k p q}(t) b(k, p, q) E(q, t)\left[k^{3} E(p, t)-k p^{2} E(k, t)\right] \frac{d p d q}{p q},
$$

where $\Delta$ indicates the subspace in the $p, q$ plane where $k, p, q$ can form a triangle, $b$ is a geometrical coefficient, depending on the triad-shape and $\theta_{k p q}$ is a function of time

$$
\theta_{k p q}(t)=\frac{1-e^{-\Lambda t}}{\Lambda}
$$

with $\Lambda=\mu_{k p q}+\nu\left(k^{2}+p^{2}+q^{2}\right), \mu_{k p q}=\mu_{k}+\mu_{p}+\mu_{q}$ and $\mu_{k}=0.5\left[\int^{k} s^{2} E(s) d s\right]^{1 / 2}$. The model details are the same as the "Unforced Case" of Ref. [38], except that the initial energy spectrum is constant and sharply truncated (as in Fig. 1). The band width is selected such that the initial band contains 100 discrete wave numbers, in order to produce correctly both local and non-local triad interactions.

The energy evolution for short times is shown in Fig. 3(a). Similar to the DNS results, there are discontinuities in the spectral slope at the boundaries of each band. Also the local time-scaling exponent $n(k, t)$ is shown in Fig. 3(b) at different time instants. The scaling exponents are staircase-shaped as expected. Comparing to Fig. 2(c), we see that the behaviour is very similar to the DNS results, therefore qualititatively the results show that EDQNM correctly captures the staircase scaling. However, the large $n$ behavior differs for the two cases (for EDQNM the scaling exponent is slightly greater than the theoretical values (horizontal lines) whereas for DNS it is slightly less than the theoretical values). Possibly this difference is due to higher-order terms neglected in Proposition 1, or the direct influence of viscous damping, more strongly present in the DNS than in the closure integration.

The advantage with closures such as the EDQNM model is that we solve directly equations for the ensemble average, so that we should be able, in principle, to understand the behavior of the solution from the equations we integrate. We will analyze the structure of the EDQNM model to understand the origin of the staircase scaling on the level of this closure, which is not confined to a single triad such as the analysis in the foregoing section supposed.

Initially, all the energy is confined in "band 0", as illustrated in Fig. 1, and we call its value $E_{0}$. Let $k$ be contained in "band 1". The energy density in this empty band, is 
governed (ignoring viscosity) by the equation

$$
\frac{\partial E_{1}}{\partial t} \approx \iint_{\Delta ; p, q<k_{0}} \theta_{k p q}(t) k^{3} b(k, p, q) E_{0}^{2} \frac{d p d q}{p q},
$$

where the only contributions are from $p, q<k_{0}$, where $E(p)=E(q)=E_{0}$. Since all interactions are restricted to $p, q<k_{0}$, the triad condition $\boldsymbol{k}=\boldsymbol{p}+\boldsymbol{q}$ will not allow interactions with modes beyond $2 k_{0}$. If the integrand would be constant in time, the energy in band 1 would grow proportional to $t$, but in the simulations and the theoretical analysis a $t^{2}$ dependence is observed. Analyzing the integrand, it is observed that it is the timescale $\theta_{k p q}$ which is time-dependent. Using a Taylor expansion, for small $t$ Eq. (7) writes

$$
\theta_{k p q}(t) \approx t
$$

so that the integrand is proportional to $t$ and the evolution of $E_{1}$,

$$
\frac{\partial E_{1}}{\partial t} \sim E_{0}^{2} t
$$

yielding a time-dependence for $E_{1}$ proportional to $t^{2}$. The exact shape of the triad-interaction time $\theta_{k p q}$ is thus important to reproduce the correct stair-case scaling.

We note that this analysis can also be extended to higher bands to obtain staircase time scalings. For example, the energy transfer to band 2 can be either the case that two wave vectors are in band 0 and 1 respectively (denoted as $\Delta_{1}$ ), or the case that two wave vectors are both in band 1 (denoted as $\Delta_{2}$ ). Therefore in short time we can write

$$
\frac{\partial E_{2}}{\partial t} \approx \iint_{\Delta_{1}} \theta_{k p q}(t) k^{3} b(k, p, q) E_{0} E_{1} \frac{d p d q}{p q}+\iint_{\Delta_{2}} \theta_{k p q}(t) k^{3} b(k, p, q) E_{1}^{2} \frac{d p d q}{p q} .
$$

Using the fact that $E_{0} \sim t^{0}$ and $E_{1} \sim t^{2}$ immediately yields a time-dependence for $E_{2}$ proportional to $t^{4}$. For higher bands the derivations are analogous.

\section{CONCLUDING REMARKS}

In the present contribution we illustrated that a sharply truncated initial spectrum leads to staircase time scalings of the small-scale response. This phenomenon is verified by DNS and EDQNM respectively. The origin of this scaling behaviour is the triadic interaction underlying the Navier-Stokes nonlinearity. 
In many cases, the spectral properties of the Navier-Stokes equations can be reproduced by a diffusion of energy in wavenumber space. The classical Leith closure [53] represents this phenomenology using a simple model-equation for the nonlinear energy transfer. The present phenomenon cannot be captured by such a model. Also the Heisenberg closure [54], which models nonlocal interactions cannot represent this, since it does not take into account the triadic nature of the nonlocal interactions. Indeed, both closures can be derived as limits of degenerate triad interactions, as was illustrated in Refs. [55, 56].

The present investigation shows how irregularities in the initial conditions can be transferred to higher wavenumbers. These irregularities are therefore not due to a nonphysical transfer mechanism, but they reflect the shape of the initial conditions.

\section{ACKNOWLEDGEMENT}

We are grateful to Liang Shao for discussions. This work is supported by the National Natural Science Foundation of China (Grant No.s 11572025, 11772032, 51420105008).

[1] A. N. Kolmogorov. The local structure of turbulence in incompressible viscous fluid for very large reynolds number. Proceedings: Mathematical and Physical Sciences, 30:301, 1941.

[2] H. L. Grant, R. W. Stewart, and A. Moilliet. Turbulence spectra from a tidal channel. Journal of Fluid Mechanics, 12(2):241-268, 1962.

[3] T. Ishihara, T. Gotoh, and Y. Kaneda. Study of high-Reynolds number isotropic turbulence by direct numerical simulation. Annual Review of Fluid Mechanics, 41:165-180, 2009.

[4] U. Frisch. Turbulence. The legacy of A. N. Kolmogorov. Cambridge University Press, 1995.

[5] J. C. Vassilicos. Dissipation in turbulent flows. Annual Review of Fluid Mechanics, 47:95-114, 2015.

[6] W. J. T. Bos and R. Rubinstein. Dissipation in unsteady turbulence. Physical Review Fluids, 2:022601, 2017.

[7] M. E. Brachet, M. Meneguzzi, A. Vincent, H. Politano, and P. L. Sulem. Numerical evidence of smooth self-similar dynamics and possibility of subsequent collapse for three-dimensional ideal flows. Physics of Fluids A: Fluid Dynamics, 4(12):2845-2854, 1992. 
[8] C. Cichowlas and M. E. Brachet. Evolution of complex singularities in Kida-Pelz and TaylorGreen inviscid flows. Fluid Dynamics Research, 36(4-6):239, 2005.

[9] C. Connaughton and S. Nazarenko. Warm cascades and anomalous scaling in a diffusion model of turbulence. Physical review letters, 92(4):044501, 2004.

[10] W. J. T. Bos, C. Connaughton, and F. Godeferd. Developing homogeneous isotropic turbulence. Physica D Nonlinear Phenomena, 241(3):232-236, 2012.

[11] S. Galtier, S. V. Nazarenko, A. C. Newell, and A. Pouquet. A weak turbulence theory for incompressible magnetohydrodynamics. Journal of Plasma Physics, 63(5):447-488, 2000.

[12] C. Connaughton, A. C. Newell, and Y. Pomeau. Non-stationary spectra of local wave turbulence. Physica D: Nonlinear Phenomena, 184(1-4):64-85, 2003.

[13] S. Thalabard, S. Nazarenko, S. Galtier, and S. Medvedev. Anomalous spectral laws in differential models of turbulence. Journal of Physics A: Mathematical and Theoretical, 48(28):285501, 2015.

[14] P. Constantin and A. Majda. The Beltrami spectrum for incompressible fluid flows. Commun. Math. Phys., 115:435-456, 1988.

[15] C. Cambon and L. Jacquin. Spectral approach to non-isotropic turbulence subjected to rotation. Journal of Fluid Mechanics, 202:295-317, 1989.

[16] F. Waleffe. The nature of triad interactions in homogeneous turbulence. Physics of Fluids A, $4(2): 350-363,1992$.

[17] A. Alexakis. Helically decomposed turbulence. Journal of Fluid Mechanics, 812:752-770, 2017.

[18] A. Briard, L. Biferale, and T. Gomez. Closure theory for the split energy-helicity cascades in homogeneous isotropic homochiral turbulence. Physical Review Fluids, 2(10):102602, 2017.

[19] L. Fang, L. Shao, J. P. Bertoglio, G. Cui, C. Xu, and Z. Zhang. An improved velocity increment model based on Kolmogorov equation of filtered velocity. Physics of Fluids, 21(6):065108, 2009.

[20] R.S. Rogallo. Numerical experiments in homogeneous turbulence. NASA TM 81315, 1981.

[21] M.K. Verma, A. Ayyer, O. Debliquy, S. Kumar, and A.V. Chandra. Local shell-to-shell energy transfer via nonlocal interactions in fluid turbulence. Pramana, 65(2):297, 2005.

[22] A. Alexakis, P.D. Mininni, and A. Pouquet. Imprint of large-scale flows on turbulence. Physical review letters, 95(26):264503, 2005. 
[23] S. A. Orszag. Analytical theories of turbulence. Journal of Fluid Mechanics, 41(2):363-386, 1970.

[24] M. Lesieur. Turbulence in Fluids. Kluwer Academic, Dordrecht, 1997.

[25] C. E. Leith. Diffusion approximation to inertial energy transfer in isotropic turbulence. Physics of Fluids, 10:1409-1416, 1967.

[26] W. Heisenberg. Zur statistischen theorie der turbulenz. Z. Physics, 124:628-657, 1948.

[27] T. T. Clark, R. Rubinstein, and J. Weinstock. Reassessment of the classical turbulence closures: the Leith diffusion model. Journal of Turbulence, (10):N35, 2009.

[28] R. Rubinstein and T. T. Clark. A generalized Heisenberg model for turbulent spectral dynamics. Theoretical and Computational Fluid Dynamics, 17(4):249-272, 2004.

[29] A. N. Kolmogorov. The local structure of turbulence in incompressible viscous fluid for very large reynolds number. Proceedings: Mathematical and Physical Sciences, 30:301, 1941.

[30] H. L. Grant, R. W. Stewart, and A. Moilliet. Turbulence spectra from a tidal channel. Journal of Fluid Mechanics, 12(2):241-268, 1962.

[31] T. Ishihara, T. Gotoh, and Y. Kaneda. Study of high-Reynolds number isotropic turbulence by direct numerical simulation. Annual Review of Fluid Mechanics, 41:165-180, 2009.

[32] U. Frisch. Turbulence. The legacy of A. N. Kolmogorov. Cambridge University Press, 1995.

[33] J. C. Vassilicos. Dissipation in turbulent flows. Annual Review of Fluid Mechanics, 47:95-114, 2015.

[34] W. J. T. Bos and R. Rubinstein. Dissipation in unsteady turbulence. Physical Review Fluids, 2:022601, 2017.

[35] M. E. Brachet, M. Meneguzzi, A. Vincent, H. Politano, and P. L. Sulem. Numerical evidence of smooth self-similar dynamics and possibility of subsequent collapse for three-dimensional ideal flows. Physics of Fluids A: Fluid Dynamics, 4(12):2845-2854, 1992.

[36] C. Cichowlas and M. E. Brachet. Evolution of complex singularities in Kida-Pelz and TaylorGreen inviscid flows. Fluid Dynamics Research, 36(4-6):239, 2005.

[37] C. Connaughton and S. Nazarenko. Warm cascades and anomalous scaling in a diffusion model of turbulence. Physical review letters, 92(4):044501, 2004.

[38] W. J. T. Bos, C. Connaughton, and F. Godeferd. Developing homogeneous isotropic turbulence. Physica D Nonlinear Phenomena, 241(3):232-236, 2012. 
[39] S. Galtier, S. V. Nazarenko, A. C. Newell, and A. Pouquet. A weak turbulence theory for incompressible magnetohydrodynamics. Journal of Plasma Physics, 63(5):447-488, 2000.

[40] C. Connaughton, A. C. Newell, and Y. Pomeau. Non-stationary spectra of local wave turbulence. Physica D: Nonlinear Phenomena, 184(1-4):64-85, 2003.

[41] S. Thalabard, S. Nazarenko, S. Galtier, and S. Medvedev. Anomalous spectral laws in differential models of turbulence. Journal of Physics A: Mathematical and Theoretical, 48(28):285501, 2015.

[42] P. Constantin and A. Majda. The Beltrami spectrum for incompressible fluid flows. Commun. Math. Phys., 115:435-456, 1988.

[43] C. Cambon and L. Jacquin. Spectral approach to non-isotropic turbulence subjected to rotation. Journal of Fluid Mechanics, 202:295-317, 1989.

[44] F. Waleffe. The nature of triad interactions in homogeneous turbulence. Physics of Fluids A, 4(2):350-363, 1992.

[45] A. Alexakis. Helically decomposed turbulence. Journal of Fluid Mechanics, 812:752-770, 2017.

[46] A. Briard, L. Biferale, and T. Gomez. Closure theory for the split energy-helicity cascades in homogeneous isotropic homochiral turbulence. Physical Review Fluids, 2(10):102602, 2017.

[47] L. Fang, L. Shao, J. P. Bertoglio, G. Cui, C. Xu, and Z. Zhang. An improved velocity increment model based on Kolmogorov equation of filtered velocity. Physics of Fluids, 21(6):065108, 2009.

[48] R.S. Rogallo. Numerical experiments in homogeneous turbulence. NASA TM 81315, 1981.

[49] M.K. Verma, A. Ayyer, O. Debliquy, S. Kumar, and A.V. Chandra. Local shell-to-shell energy transfer via nonlocal interactions in fluid turbulence. Pramana, 65(2):297, 2005.

[50] A. Alexakis, P.D. Mininni, and A. Pouquet. Imprint of large-scale flows on turbulence. Physical review letters, 95(26):264503, 2005.

[51] S. A. Orszag. Analytical theories of turbulence. Journal of Fluid Mechanics, 41(2):363-386, 1970.

[52] M. Lesieur. Turbulence in Fluids. Kluwer Academic, Dordrecht, 1997.

[53] C. E. Leith. Diffusion approximation to inertial energy transfer in isotropic turbulence. Physics of Fluids, 10:1409-1416, 1967.

[54] W. Heisenberg. Zur statistischen theorie der turbulenz. Z. Physics, 124:628-657, 1948. 
[55] T. T. Clark, R. Rubinstein, and J. Weinstock. Reassessment of the classical turbulence closures: the Leith diffusion model. Journal of Turbulence, (10):N35, 2009.

[56] R. Rubinstein and T. T. Clark. A generalized Heisenberg model for turbulent spectral dynamics. Theoretical and Computational Fluid Dynamics, 17(4):249-272, 2004. 